\title{
The Application of Irap Markers in the Breeding of Papaya (Carica Papaya L.)
}

\author{
Kamaludin Rashid', Rofina Yasmin Othman², Bathusha Shareef Bin Kader Shaik Ali², \\ Yusmin Mohd Yusof ${ }^{1}$ and Arash Nezhadahmadi* \\ ${ }^{1}$ Centre for Foundation Studies in Science, University of Malaya, 50603, Kuala Lumpur, Malaysia \\ ${ }^{2}$ Institute of Biological Sciences, Faculty of Science, University of Malaya, 50603, Kuala Lumpur, Malaysia; \\ nezhadahmadi.arash@gmail.com
}

\begin{abstract}
Papaya is an important medicinal crop which is used in industries and other healthcare products. This study was conducted to facilitate the selection of Papaya Ring Spot Virus (PRSV) resistant papayas in breeding programs by DNA fingerprinting method. By using Inter-Retrotransposons Amplified Polymorphism molecular markers, 5 out of 45 possible primer combinations (28 IRAP primers combination and $R T$ primers) showed significant polymorphism in DNA banding patterns that determined parental-progeny relations and classified progenies based on their resistance towards PRSV. Primers combination of LTR 6149 and LTR 6149 had the highest degree of polymorphism which was 75\% of polymorphic bands. It is observed that only 1 out of 5 combinations of primers demonstrated DNA banding patterns similar to the previous study by Malaysian Agriculture and Research Development Institute (MARDI). Phylogenetic trees were constructed to detect the relation of papaya samples, and those which based on primers combination of LTR 6150 and Nikita showed resistant progenies with resistant parent. Furthermore, progenies from $L 90$ were found to be closely related to the parent Tainung (T5) whilst progenies from $L 13$ and $L 33$ were closely related to another parent, Eksotika (E6). L90 with symptom-free fruits was the most tolerant followed by L33 and L13. So, the best primers in terms of inheritance pattern based on the PRSV resistance trait (LTR 6150 and Nikita primers) were suggested to be further applied in Molecular Assisted Breeding (MAB) or Molecular Assisted Selection (MAS) for papaya breeding programs.
\end{abstract}

Keywords: Breeding, Retrotransposon, IRAP Markers, Carica Papaya, Papaya

\section{Introduction}

Papaya is a herbaceous fruit tree grown in most part of the world. It belongs to Brassicales and Caricaceae family and shares the same ancestor as Arabidopsis. Carica papaya (paw-paw or papaw) is an angiosperm, a tropical dicot, and the most important plant in terms of nutritional value based on the percentage of Vitamin C, potassium, Vitamin A, niacin, riboflavin, iron, thiamine, calcium, and fibre. The fruits, leaves, stems and roots of papaya are used in medical applications, involving papain production, a precious proteolytic enzyme ${ }^{1}$. The small genome size of papaya and rapid production of fruits have made it a potential model organism for fruit-producing crops $^{2}$. The production of this economically imperative fruit is restricted by the harmful illness caused by Papaya Ring spot Virus (PRSV) and the perishable and fragile nature of the fruit limit largescale exportation ${ }^{3}$. The use of molecular markers for superior cultivar selection has been exhibited in a vast number of plant species ${ }^{4,5,6,7}$. Molecular markers such as retrotransposons are promising strategies in assisting selection for desirable character ${ }^{8}$. Retrotransposons may play a crucial functions such as reactions to environmental cues, identification of continuous phenotypic traits and gene regulation ${ }^{9}$. Retrotransposons possess different benefits as molecular markers in which their dispersion and abundance can produce various marker bands, the

${ }^{*}$ Author for correspondence 
pattern which has a high polymorphism level because of transposition activity ${ }^{10,11}$. The terminal sequences of long-terminal repeat $(L T R)$ retrotransposons are a source of strong molecular markers for linkage mapping and biodiversity studies. RNA-mediated class I retrotransposons, or Long Terminal Repeat (LTR) retrotransposons are mobile genetic factors that transpose through reverse transcription of an RNA intermediate. The highest studied of LTR retrotransposons group is the Ty1-copia, named after the best characterised factors in Drosophila melanogaster (copia) and Saccharomyces cerevisiae (Ty1). It is now well known that retrotransposons of the Ty1copia group are ubiquitous in plants ${ }^{12,13,14}$ and constitute a significant fraction of all plant genomes. Forty per cent or more of the total genome may consist of retrotransposon sequences, based on estimates using the reverse transcriptase domain of Ty1-copia as a probe in broad (or field) bean, Vicia faba ${ }^{15,16}$. To distinguish polymorphisms for insertion of retrotransposon, the recently developed marker systems rely on Polymerase Chain Reaction (PCR) amplification between some components of the flanking genomic DNA and an $L T R^{10}$. This method is known as Inter Retrotransposons Amplified Polymorphisms-Polymerase Chain Reaction or IRAPPCR. IRAP is a dominant, multiplex marker system that examines variation in retrotransposon insertion sites. It is a PCR (Polymerase Chain Reaction) based marker technique that uses one or two primers pointing outwards from an $L T R$, and thus amplifies the tract of DNA among two nearby retrotransposons. The IRAP bands are produced from two nearby LTRs with the use of outward-facing primers annealing to $L T R$ target sequences ${ }^{10}$. IRAP fragments between two retrotransposons are produced by PCR which can also be used for unravelling the evolutionary history of retrotransposons insertions in plants ${ }^{17}$. The IRAP fingerprinting patterns can also be applied in different applications, involving estimation of population structure and genetic diversity, detection of essential derivation, recombination mapping and marker assisted selection ${ }^{10}$. In this study, IRAP method in DNA fingerprinting technique was applied to identify molecular markers which successfully classify papaya samples according to their resistance towards Papaya Ringspot Virus's infection. Moreover, screening of IRAP primers which produce polymorphic banding patterns was conducted, phylogenetic trees were constructed, and pattern of inheritance in papaya samples were studied.

\section{Materials and Methods}

\subsection{Plant Materials}

Leaves of Carica papaya L. from Tainung 5, Eksotika 6 varieties and their hybrids were collected from Malaysian Agriculture Research and Development Institute (MARDI). The leaves of these plants were used as a source of genomic DNA (gDNA) for PCR amplification of IRAP bands. Prior to plant DNA extraction, following solutions were prepared: Extraction buffer (consisting of 5\% hexadecyl trim ethyl ammonium bromide (CTAB), $1.4 \mathrm{M}$ $\mathrm{NaCl}, 0.4 \%$ 2-mercaptoethanol, $20 \mathrm{mM}$ EDTA, $100 \mathrm{mM}$ Tris-HCL, pH 8.0), TE buffer (10mM Tris-HCL, pH8, $1 \mathrm{mM}$ EDTA), and washing solution. Extraction protocol involved a modified CTAB procedure according to Doyle and Doyle ${ }^{18}$. Approximately $3.0 \mathrm{~g}$ of young papaya leaf (Carica papaya L.) was grounded using mortar and pestle with liquid nitrogen. The frozen powder was then poured into a polypropylene tube containing $15 \mathrm{ml}$ of preheated Extraction Buffer ( $\mathrm{pH} 8$ ). The extraction buffer and plant frozen powder were combined evenly and incubated at temperature $60^{\circ} \mathrm{C}$ for 60 minutes with occasional gentle inverted mixing every 10 minutes. After that, the mixture was cooled at room temperature and equal volume of chloroform was added. The mixture was then centrifuged at $5000 \mathrm{rpm}$ for 10 minutes at $25^{\circ} \mathrm{C}$. DNA was precipitated by adding 0.6 volume of ice-cold isopropanol and stored at $-20^{\circ} \mathrm{C}$ for 30 minutes. Precipitated DNA was centrifuged at $5000 \mathrm{rpm}$ for 10 minutes at $4^{\circ} \mathrm{C}$. Supernatant was decanted carefully and pellet washed with $20 \mathrm{ml}$ of washing solution. The pellet was then agitated gently and centrifuged at 5000 rpm for 10 minutes at $4^{\circ} \mathrm{C}$. After that, 2 volume of $96 \%$ ethanol was added, mixed by inversion, stored at $-20^{\circ} \mathrm{C}$ for 1 hour and later centrifuged at $5000 \mathrm{rpm}$ for 10 minutes at $4^{\circ} \mathrm{C}$. Next, the pellet was being washed finally with $70 \%$ icecooled ethanol and centrifuged at $5000 \mathrm{rpm}$ for 10 minutes at $4^{\circ} \mathrm{C}$. The pellet was then dried in a speed vacuum for 20 minutes before finally being dissolved in $1 \mathrm{ml}$ of deionised water. After the DNA dissolved in the water, the tube was kept at $-20^{\circ} \mathrm{C}$ as stock for further analysis. The purity of the nucleic acid samples was determined by calculating the ratio between the optical density readings at $260 \mathrm{~nm}$ and $280 \mathrm{~nm}$. For estimating DNA concentration, spectrophotometric measurement of UV absorbance at $260 \mathrm{~nm}$ using Eppendorf's Biophotometer machine was carried out. An optical density of 1.0 at $260 \mathrm{~nm}$ is equivalent to $50 \mathrm{mg} / \mathrm{mL}$ of double-stranded DNA. Prior 
to Biophotometer readings, all DNA samples were diluted 100-1000 times using desterilized water. The IRAP method was conducted as explained by Kalendar et al. ${ }^{19}$, Kalendar et al. ${ }^{20}$ and Boyko et al. ${ }^{21}$. The Sukkula and Nikita LTR primers were designed facing outward from the Sukkula LTR and Nikita LTR, respectively. The LTR 6149, LTR 6150, 5' LTR1 and 5' LTR2 were designed facing outward from the $5^{\prime} L T R$ region of the BARE-1a retrotransposons sequence whereas the $3^{\prime} L T R$ was designed facing outward from the 3' ends of the BARE-1a LTR. The IRAP primers were designed from the long terminal repeats (LTR) of the BARE-1 retrotransposon and the internal region of reverse transcriptase (RT) of papaya Ty1-copia-like retrotransposons. The reverse primer LTR 6150 was complementary to bases $418-439$ of the 5' LTR of the BARE-1a sequence (accession Z17327, 309-2137), and the forward primer LTR 6149 matched bases 1993-2012 of the 5' LTR of the BARE-1a sequence (accession Z17327). The primers location corresponded to the preserved stretches of the $B A R E-1$ LTR, especially at the 3 ' ends of the primers, based on initial alignments. The $5^{\prime}$ LTR 1 primer matched to bases 36369-36394 of the 5' LTR of the BARE-1 sequence (accession AF254799, 34586-36407) whereas the 5' LTR2 primer was complementary to bases $310-331$ of the $5^{\prime} L T R$ of the BARE-1a sequence (accession Z17327). The primers location corresponded to preserved stretches of the 5 ' ends of the BARE-1 LTR. The 3' LTR primer was complementary to bases 14971-14994 of the 3' LTR of the BARE-1 retrotransposon (accession AF254799, 14967-16796). The Sukkula LTR primer matched bases 4935-4959 of the Sukkula LTR-1 (accession AF254799, 6245-11204) and the Nikita LTR primer matched bases 1623-1645 of the Nikita LTR-1 (accession AF254799, 38443-40171). For IRAP-PCR, the total genomic DNA of papaya samples was diluted with sterile $\mathrm{dH} 2 \mathrm{O}$ to $100 \mathrm{ng} / \mu \mathrm{l}$ concentration. After the IRAPPCR reaction, the IRAP-PCR products were analysed by electrophoresis on $1.6 \%(\mathrm{w} / \mathrm{v})$ agarose and determined by ethidium bromide staining. The agarose gel was analysed with the use of Quantity One version 4 for Windows (Bio-Rad, USA).

\section{Results}

All 16 papaya samples showed optical density (OD) readings that ranged between 1.7 and 1.9 while DNA concentration ranged between $0.5 \mu \mathrm{g} / \mu \mathrm{L}$ and $1.5 \mu \mathrm{g} / \mu \mathrm{L}$. All samples showed presence of intact DNA bands in electrophoresis gel though there were some indications of DNA degradation in some bands. Optical density (OD) readings showed sufficient level of purity to be used in IRAP-PCR. DNA concentration of samples obtained ranges from low to highly concentrated DNA. Figure 1 shows IRAP-PCR DNA fingerprinting using primer combinations of LTR 6150 and $5^{\prime}$ LTR. The first lane marked the DNA molecules size (100bp marker). The arrows indicate bands that are present in either one of the parents only. Lane 2-3 represents parental papayas (E6 and T5) and lane 4-17 represents 14 hybrid papayas from 4 lines (L13-L90). The primers combination produced 20 bands whereby 11 were polymorphic (55\% polymorphism). Figure 2 demonstrates IRAP-PCR using primers

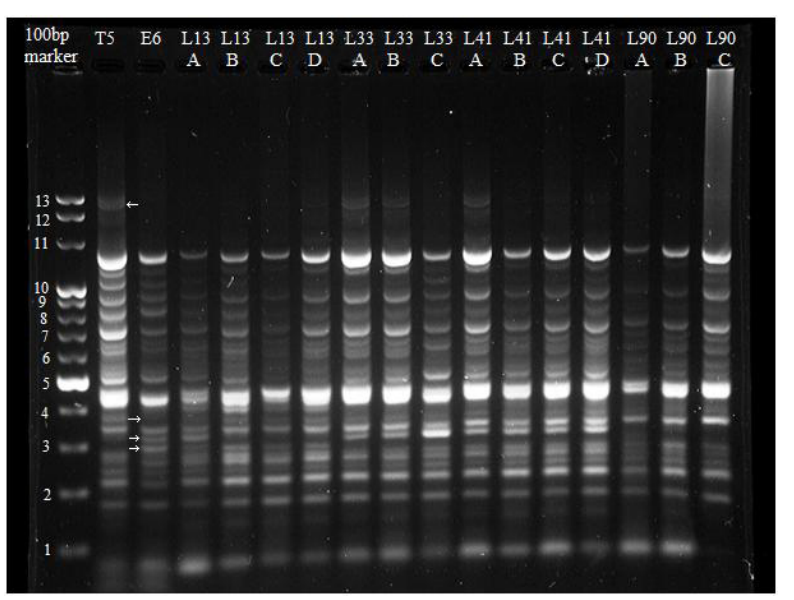

Figure 1. IRAP-PCR using primer combination of LTR 6150 and 5'LTR1

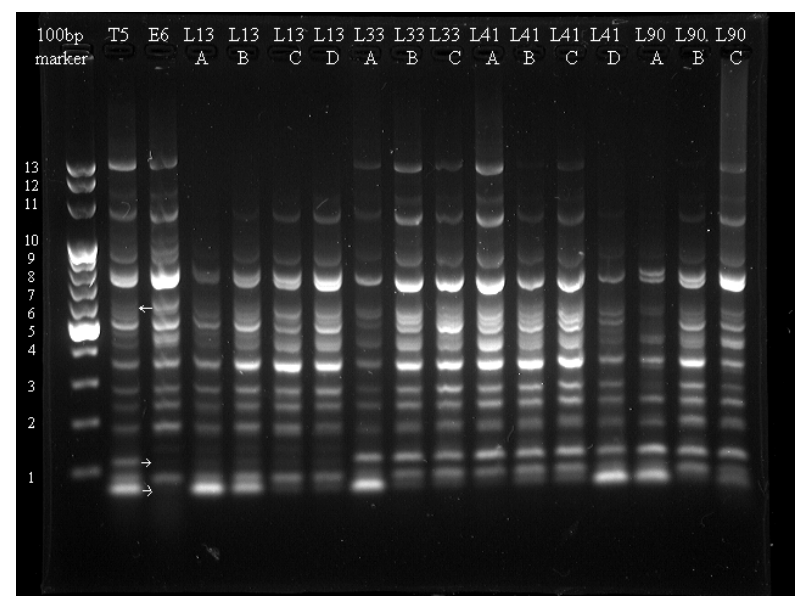

Figure 2. IRAP-PCR using primer combination of $L T R$ 6150 and Nikita LTR 
combination of LTR 6150 and Nikita LTR. It was observed that some bands were highly expressed which indicated their strong responses to the marker, however, others responded weakly as their bands were thin. The molecular weights of the $100 \mathrm{bp}$ DNA ladder were $1=100 \mathrm{bp}$, $5=500 \mathrm{bp}, 7=700 \mathrm{bp}, 10=1000 \mathrm{bp}$, and $13=2500 \mathrm{bp}$. From 16 bands scored, eight were polymorphic bands (50\% polymorphism). Figure 3 shows IRAP-PCR using primer LTR6149. Though it produced rather thin bands, out of 12 bands obtained, nine were polymorphic ( $75 \%$ polymorphism). According to Figure 4, the results were achieved with the use of primer combinations of LTR 6150 and $R T$ (reverse). The primers combination has a polymorphism percentage of $60 \%$ whereby out of 15 bands scored, nine

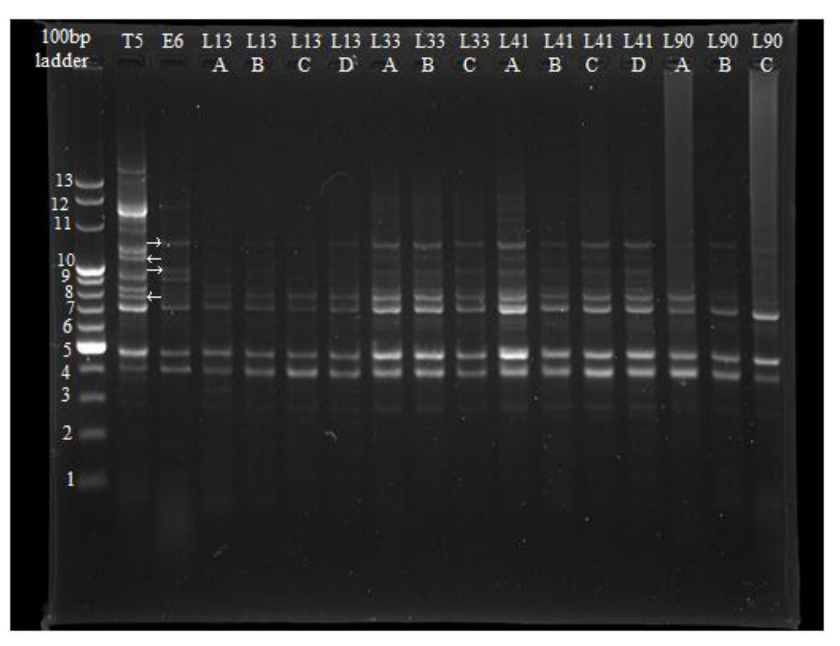

Figure 3. IRAP-PCR using primer LTR 6149

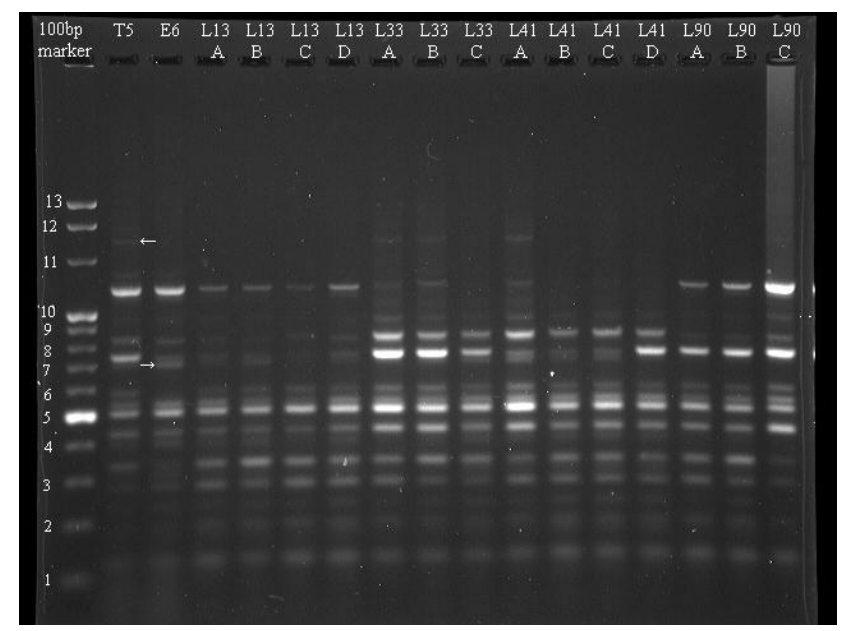

Figure 4. IRAP-PCR using primer combination of LTR 6150 and $R T$ (reverse) were polymorphic bands. Based on Figure 5, IRAP-PCR using $R T$ (forward and reverse) primers combination produced 18 bands and seven of them were polymorphic (39\% polymorphism). The level of polymorphism of IRAP products in Carica papaya L. was high between $39 \%$ and $75.0 \%$ (Table 1). In total, about half of IRAP products had significantly polymorphic bands with $54 \%$ degree of polymorphism. Combination of IRAP primers with the lowest degree of polymorphism was $R T$ Forward \& $R T$ Reverse with $39 \%$ of polymorphic bands while primers combination of LTR 6149 and LTR 6149 had the highest degree of polymorphism which was $75 \%$ of polymorphic bands. The smallest band was 70 base pairs long and the biggest band was 1500 base pairs long. Both

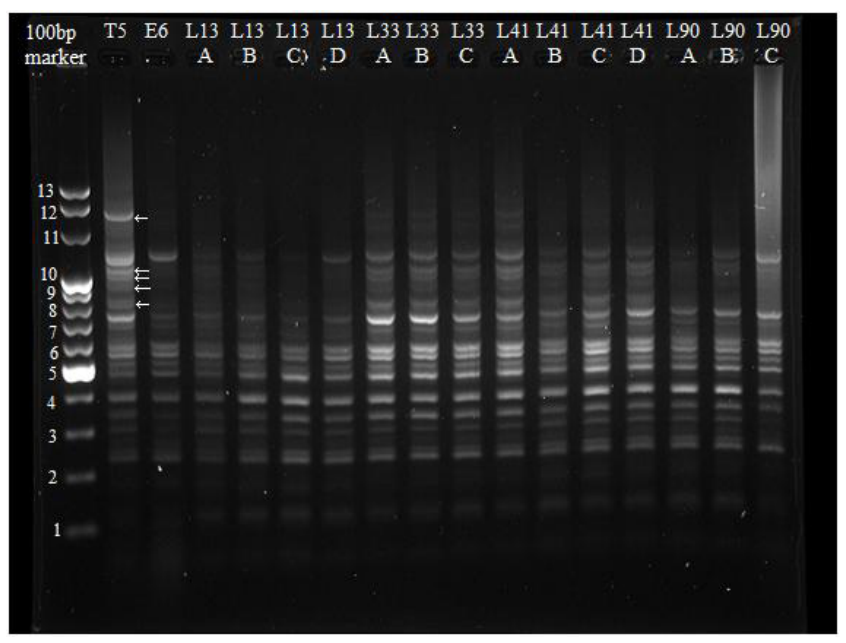

Figure 5. IRAP-PCR using RT (forward and reverse) primers combination

Table 1. Polymorphism degree of IRAP markers in Carica papaya

\begin{tabular}{lccc}
\hline $\begin{array}{l}\text { Primers } \\
\text { combination }\end{array}$ & $\begin{array}{c}\text { Total } \\
\text { bands }\end{array}$ & $\begin{array}{c}\text { Polymorphic } \\
\text { bands }\end{array}$ & $\begin{array}{c}\text { Degree of } \\
\text { polymorphism } \\
(\%)\end{array}$ \\
\hline $\begin{array}{l}\text { LTR 6150 \& 5'LTR1 } \\
\text { LTR 6150 \& }\end{array}$ & 20 & 11 & $55 \%$ \\
$\begin{array}{l}\text { Nikita LTR } \\
\text { LTR 6149 \& }\end{array}$ & 16 & 8 & $50 \%$ \\
$\begin{array}{l}\text { LTR 6149 } \\
\text { LTR 6150 \& } \\
\text { RT (reverse) }\end{array}$ & 12 & 9 & $75 \%$ \\
$\begin{array}{l}\text { RT (forward \& } \\
\text { reverse) }\end{array}$ & 18 & 9 & $60 \%$ \\
\hline Total & 81 & 44 & $39 \%$ \\
\hline
\end{tabular}


T5 and E6 samples produced polymorphic bands which showed inter-specific banding patterns. There were in total 81 polymorphic bands between the samples, some shorter fragments were amplified from most lines, proposing preservation of the internal organization of retro elements parts. These outcomes showed the transferable nature of the retrotransposon-based marker system ${ }^{22}$. All 6 universal primers amplified different fragments of defined sizes from papaya gDNA under the PCR conditions used, with different level of polymorphisms (Table 1). The bands number and polymorphism level were presented through percentage of polymorphic bands also in a way reflected the copy number and distribution of LTR retrotransposons in papaya. This study which used molecular techniques provided support of the discoveries of LTR retrotransposons copy number and distribution which used cytological techniques.

\subsection{Phylogenetic Tree Construction}

In this study, all phylogenetic trees were constructed using Dollop program from PHYLIP (PHYLogeny Inference Package) software (version 3.69). These program and method were chosen as they generated fully-resolved phylogenetic trees, produced results quickly and were easy to use. The method chosen in this study was polymorphism parsimony. This method was first used by Felsenstein ${ }^{23}$. Phylogenetic trees were constructed from tables involving scores for absence or presence of bands of particular mobility in accessions. Only bands that were detectable were taken into account for scoring. A value of 1 defined the presence of a band of a particular mobility compared to a DNA ladder and a value of 0 defined the absence of that particular band. According to Figure 6, this phylogenetic

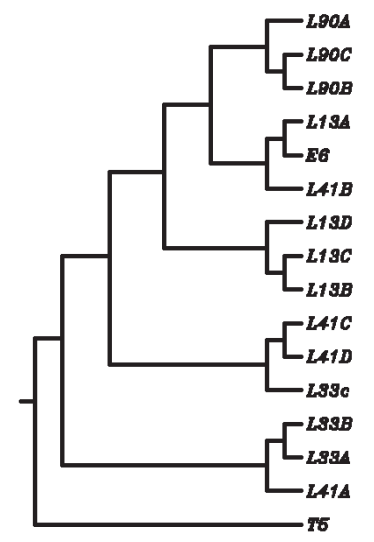

Figure 6. Phylogenetic tree of Carica papaya L. based on LTR 6150 and 5'LTR1 primers tree has five (5) subgroups and each subgroup has 3 samples. T5 which is the out group is closely related to sample $L 41 A$ followed by samples $L 33 A$ and $L 33 B$. In the second subgroup from top, another parental sample, E6 is closely related to sample $L 13 \mathrm{~A}$ followed by sample $L 41 B$. Figure 7 demonstrates the phylogenetic tree which is divided into two major groups which are further divided into 3 and 2 subgroups each. Outside of these two major groups there is a subgroup which is closely related to the out group (sample T5). The subgroup is made of samples $L 90 A$ and $L 41 D$. Another parental sample E6 is closely related to samples $L 13 C$ and $L 13 D$. Figure 8 shows that the phylogenetic tree that is divided into two major groups. Each major groups is further divided into three subgroups making up a total of six subgroups overall. From the topology, sample T5 formed the out group and was closely related to samples $L 13 A$ and $L 13 B$. Another

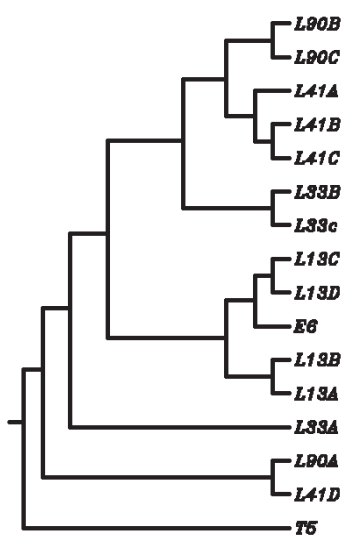

Figure 7. Phylogenetic tree of Carica papaya L. based on LTR 6150 and Nikita LTR primers

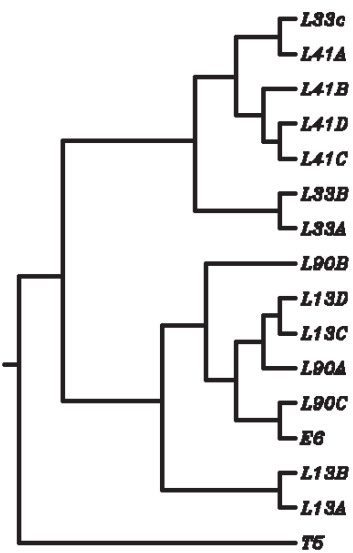

Figure 8. Phylogenetic tree of Carica papaya L. based on LTR 6149 primers 
parental sample E6 was closely related to samples $L 90 \mathrm{C}$. Figure 9 exhibits the phylogenetic tree which is divided into 2 major groups and 3 subgroups. Based on this figure, sample $T 5$ is closely related to samples $L 13 A$ and $L 13 D$. Another parental sample E6 is closely related to sample L13B. Based on Figure 10, phylogenetic tree has two subgroups. Parental sample T5 is the out group and closely related to sample L33A. Sample E6 is closely related to sample $L 41 D$. The two subgroups comprise of samples from $L 90$ and $L 13$ exclusively.

\section{Discussion}

Papaya DNA extraction was successful and all samples showed presence of intact DNA bands though there were some indication of DNA degradation in some bands.

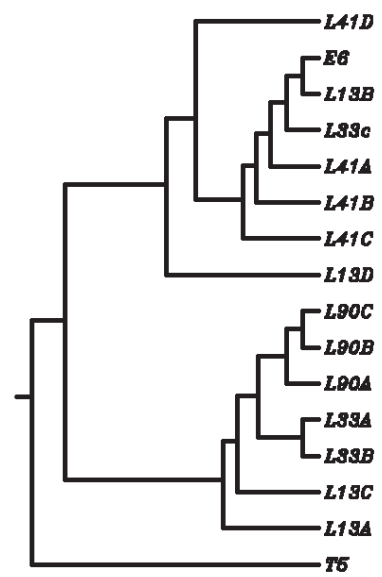

Figure 9. Phylogenetic tree of Carica papaya L. based on LTR 6150 and RT (reverse) primers

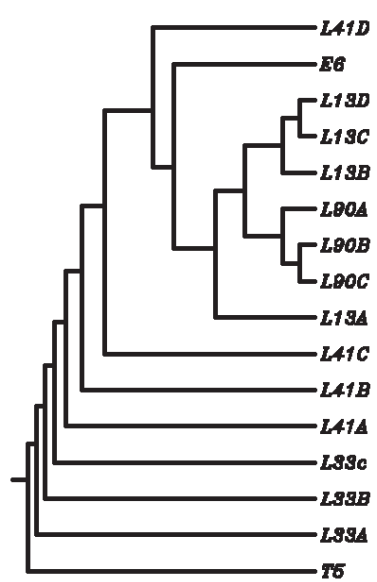

Figure 10. Phylogenetic tree of Carica papaya L. based on $R T$ (forward and reverse) primers
Optical density (OD) readings showed sufficient level of purity for use in IRAP-PCR. For use in IRAP-PCR, further purification step such as RNase treatment was not necessary. DNA concentration of samples obtained ranged from low concentration to highly concentrated DNA. Among a total of 45 possible IRAP primers combination and $R T$ primers, only 5 primers combination successfully produced significant polymorphic banding pattern results. Furthermore, the 5 primers combinations produced significant polymorphic banding patterns that discriminate between parental samples. This is important to determine parental-progeny relations and to classify progenies based on their resistance towards PRSV (resistant progenies to resistant parent and vice versa). Based on the IRAP-PCR results, the PCR bands were then scored and the data was used to construct phylogenetic trees. Phylogenetic trees were constructed as they were able to depict parental-progenies relations of the samples and thus the pattern of inheritance. Amongst all 5 phylogenetic trees constructed, phylogenetic tree based on the bands scoring of primers combination LTR 6150 and Nikita classified resistant papaya progenies with resistant parent and vice versa (Figure 7). From the phylogenetic tree, progenies from $L 90$ were found to be the closest related to parent Tainung (T5) whilst progenies from L13 and $L 33$ were found to be closely related to the other parent, Eksotika (E6) (Figure 8). This finding was the same as field trial findings by $\mathrm{Chan}^{24}$. L90 with near symptomfree fruits was the most tolerant insofar as fruit symptoms were concerned. At 12 months, when fruit symptom expression was highly severe, $L 90$ fruits had hardly any symptoms like ring spots or necrosis. L13 was found to have TSS (total soluble solids), closest fruit weight to Eksotika and had tolerance to disease only just lower than the three most tolerant lines. $L 41$ and $L 90$ would make useful parents for development of F1 hybrids in future breeding program $\mathrm{s}^{24}$. Therefore, there was a similarity between the results obtained in IRAP molecular marker studies (based on phylogenetic tree groupings) and field trial analysis of papaya parents and hybrids in MARDI breeding program. Nevertheless, selection of resistant papayas based on molecular markers was without consideration of yield and quality. Tolerance to disease in fruit crops would be rather meaningless without consideration of yield and quality. This is more in the present case where the genotypes were derived from single seed descent without prior selection pressure on yield and quality ${ }^{24}$. Most lines seemed to bear quite well under PRSV infected 
conditions. Their yield ranged from 29.5 to $41.7 \mathrm{~kg}$ /tree which was quite comparable to $35-60 \mathrm{~kg} /$ tree previously reported for PRSV tolerant 'Sinta' hybrid ${ }^{24}$. Yield and quality might be affected even though the plant is able to resist a virus infection. The other phylogenetic trees produced mix classification between resistant and susceptible papayas. Therefore, the trees lineages did not follow exactly the findings of field trial results. Probably these primers were located far from the resistant gene embedded in genome of Tainung 5 and hence did not follow its inheritance pattern. It can be deduced that the orientation and sequence of Nikita and LTR 6150 primers were strategically located near to PRSV resistant gene in the papaya genome which were being amplified through IRAP-PCR. This in turn caused Nikita and LTR 6150 banding patterns to classify papaya genotypes according to their resistance towards PRSV. To increase the chances of IRAP markers being near to the gene of interest (PRSV resistance gene), more papaya-derived IRAP markers should be designed and tested. This is because, the nearer the IRAP markers to the resistant gene, the more accurate the IRAP banding patterns which follows papayas resistance towards PRSV will be. Furthermore, with the availability of papaya whole genome sequence database and papaya repeat database such as CPR-DB, retrotransposons native LTR ends can be analysed and compared ${ }^{9}$. The use of $R T$ primers matching highly protected domains $s^{12,25,26}$ permits the detection of insertions into virtually any copia-like retrotransposons. A mix of $L T R$ and $R T$ primers can be applied to track nested insertions events whereby a retrotransposons is integrated into the LTR or internal region of another retrotransposons. Such nested patterns of insertions are common in maize and barley ${ }^{27,28}$. Methods have been improved for rapidly isolating native retrotransposons based on preserved domains within retrotransposons ${ }^{29}$. From this study, it was found that universal TY1-copia Reverse Transcriptase primers (reverse and forward primers), could be used together with other IRAP primers. Due to its location and orientation, the use of $R T$ primers with other IRAP primers would enable new papaya-specific LTR sequence to be obtained. Once cloned and sequenced, new papaya-derived IRAP primers can be designed and tested. Since Tainung 5 was resistant towards infection by PRSV, it is clear that Tainung 5 possesses PRSV resistant gene other than the transgenic papaya. Thus, it would be beneficial to study and analyse other resistant genes that were present in Tainung 5 or other wild varieties with the availability of papaya genome database. Among the reasons retrotransposons being chosen as molecular marker is the probability of retrotransposons to transpose themselves near resistance genes. IRAP-PCR products might actually contain the resistance genes being much searched for. Therefore, if the retrotransposons are located near to the resistance gene based on banding pattern which is similar to resistant plant banding pattern, there is a high probability that cloning and sequencing those very bands might reveal the resistance gene sequence itself. Last but not least, genetic transformation of plants with PRSV code protein has successfully enabled papayas 'cross protection.' Nonetheless, plants pathologists do not offer detailed explanation of the resistant mechanism which produced such results. One speculation that can be made is that the transgenic plant resistance (tobacco, papaya) which contain the virus' coat protein gene would cause the expression of both the coat protein gene by the plant's DNA and from the virus. This would then cause coat protein competition between the protein expressed from the plant and from the virus DNA to wrap up (package) the virus' genetic material. Thus, the failure to wrap up the virus' genetic material due to this competition would in turn result in the failure of the whole infection altogether and deem the plant resistant.

\section{Conclusion}

In this study, combinations of IRAP primers were screened thoroughly and the combinations which produced polymorphic banding pattern were scored. Phylogenetic trees were constructed and population segregation/pattern of inheritance were studied. IRAP markers which were able to select tolerant papayas towards Papaya Ring spot Virus infection was identified through comparison with field trial results. Relatively all primers were useful but the best combination in term of inheritance pattern based on the PRSV resistance trait were LTR 6150 and Nikita primers. Eventhough barley-derived, the IRAP markers used in this study were proven to be applicable, useful and informative to detect tolerant papaya in Malaysia breeding program. This in turn expedite the time taken to evaluate candidates for future studies and facilitate farmers in selection such as molecular assisted breeding and molecular assisted selection. More papaya samples from other genotypes could also be tested in future studies to further compare and analyse marker results with field trial results. Moreover, to ease bands scoring and comparison, bigger agarose gel should be used so that 
it can run more samples. Whenever possible, samples to be compared to each other should be run in adjacent lanes. This is crucial if high-precision comparisons are required. Large sample numbers, however, make it necessary to determine band matching between lanes which are widely separated and even among lanes derived from various gels. In these cases, proper standards have to be involved at least every four to five lanes on each gel. In most cases, molecular weight markers are applied for this target. However, one of the investigated samples may also serve as a standard, especially if it contains a set of invariable bands present in all individuals ${ }^{30}$. In this research, significant polymorphic bands were defined as bands that discriminate between parental samples. This was to classify progenies according to their closest parent. The repetitive, dispersed nature of many long terminal repeat (LTR)-retrotransposons families have been exploited for classifying progenies in Carica papaya L. hybrids. The IRAP markers distinguished the parental-progenies genomes of the papaya varieties. The IRAP markers enabled phylogenetic trees analysis of Malaysian and Taiwanese papaya varieties and their hybrids to be carried out and provided information about resistance towards papaya ring spot virus's infection. In applied work, the method based on PCR-based assay is amenable to the large-scale throughput demands of screening breeding populations and is applicable to any plant. More attention should also be given towards using LTR sequences from Ty3-gypsy retrotransposons as Ty3gypsy is found to be more abundance in papaya genome compared to Ty1-copia retrotransposons. Therefore, the probability of Ty3-gypsy retrotransposons being near to resistance genes in the papaya genome would definitely be higher than Ty1-copia. Moreover, it would be ideal if future designed markers are unique to one of the parents involved so that the banding patterns produced would definitely discriminate between parental samples. In future studies, it is recommended that primers LTR 6150 and Nikita should be tested for use in Molecular Assisted Breeding (MAB) and Molecular Assisted Selection (MAS). Hopefully, these recommended IRAP markers would prove to be useful in improving the papaya industry in Malaysia.

\section{Acknowledgment}

The authors are grateful to the University of Malaya for their support and research grant provided.

\section{References}

1. Ming, R. et al. The draft genome of the transgenic tropical fruit tree papaya (Carica papaya Linnaeus). Nature, 2008;452, 991-996.

2. Ming, R., Moore, P. H., Zee, F., Abbey, C. A., Ma, H., \& Paterson, A. H. Construction and characterization of a papaya BAC library as a foundation for molecular dissection of a tree-fruit genome. Theor Appl Genet, 2001;102, 892-899.

3. Yeh, S. D., Bau, H. J., Kung, Y. J., \& Yu, T. A. Papaya. Biotechnology in Agriculture and Forestry, 2007;60, 73-96.

4. Jefferies, S. P., King, B. J., Barr, A. R., Warner, P., Logue, S. J., \& Langridge, P. Marker-assisted backcross introgression of the $\mathrm{Yd}_{2}$ gene conferring resistance to barley yellow dwarf virus in barley. Plant Breed, 2003;122, 52-56.

5. Lecouls, A. C., Bergougnoux, V., Rubio-Cabetas, M. J., Bosselut, N., Voisin, R., Poessel, J. L., Faurobert, M., Bonnet, A., Salesses, G., Dirlewanger, E., \& Esmenjaud, D. Marker-assisted selection for the wide-spectrum resistance to root-knot nematodes conferred by the Ma gene from Myrobalan plum (Prunus cerasifera) in interspecic Prunus material. Mol Breed, 2004;13, 113-124.

6. Narayanan, N. N., Baisakh, N., Oliva, N. P., Vera Cruz, C. M., Gnanamanickam, S. S., Datta, K., \& Datta, S. K. Molecular breeding: marker-assisted selection combined with biolistic transformation for blast and bacterial blight resistance in Indica rice (cv. CO39). Mol Breed, 2004; $14,61-71$.

7. Yi, G., Lee, S. K., Hong, Y. K., Cho, Y. C., Nam, M. H., Kim, S. C., Han, S. S., Wang, G. L., Hahn, T. R., Ronald, P. C. \& Jeon, J. S. Use of Pi5(t) markers in marker-assisted selection to screen for cultivars with resistance to Magnaporthe grisea. Theor Appl Genet, 2004;109, 978-998.

8. Mohan, M., Nair, S., Bhagwat, A., Krishna, T. G., Yano, M., Bhatia, C. R., \& Sasaki, T. Genome mapping, molecular markers and marker-assisted selection in crop plants. Molecular Breeding, 1997;3, 87-103.

9. Nagarajan, N., Navajas-Perez, R., Pop, M., Alam, M., Ming, R., Paterson, A. H., \& Salzberg, S. L. Genome-Wide Analysis of Repetitive Elements in Papaya. Tropical Plant Biol, 2008;1, 191-201.

10. Kalendar, R., \& Schulman, A. H. IRAP and REMAP for retrotransposon-based genotyping and fingerprinting. Nature Protocols, 2006;1(5), 2478-2484.

11. Kumar, A., \& Bennetzen, J. L. Plant retrotransposons. Annu Rev Genet, 1999;33, 479-532.

12. Flavell, A.J.Dunbar,E., Anderson, R., Pearce, S. R., Hartley, R., \& Kumar, A. Ty1-copia group retrotransposons are ubiquitous and heterogeneous in higher plants. Nucl. Acids Res, 1992;20, 3639-3644. 
13. Flavell, A. J., Pearce, S. R., \& Kumar, A. Plant transposable elements and the genome. Curr.Op.Genet.Devel, 1994;4, 838-844.

14. Kumar, A. The adventures of the Ty1-copia group of retrotransposons in plants. Trends Genet, 1996;12, 41-43.

15. Pearce, S. R., Harrison, G., Li, D., Heslop-Harrison, J. S., Kumar, A., \& Flavell, A. J. The Ty1-copia group retrotransposons in Vicia species: copy number, sequence heterogeneity and chromosomal localisation. Mol. Gen.Genet, 1996a;250, 305-315.

16. Pearce, S. R. Harrison, G., Heslop-Harrison, J. S., Flavell, A. J., \& Kumar, A. The Ty1-copia group retrotransposons of Allium cepa are distributed throughout the chromosomes but are enriched in the telomeric heterochromatin. Chromosome Res, 1996b;357-364.

17. Price, Z., Dumortier, F., MacDonald, D. W., \& Mayes, S. Characterization of copia-like retrotransposons in oil palm (Elaeis guineensis Jacq.). Theor Appl Genet, 2002;104, 860-867.

18. Doyle, J. J., \& Doyle, J. L. A rapid total DNA preparation procedure for fresh plant tissue. Focus, 1990;12, 13-15.

19. Kalendar, R., Grob, T., Regina, M., Suoniemi, A., \& Schulman, A. H. IRAP and REMAP: two new retrotransposons-based DNA fingerprinting techniques. Theor Appl Genet, 1999;98, 704-711.

20. Kalendar, R., Tanskanen, J., Immonen, S., Novo, E., \& Schulman, A. H. Genome evolution of wild barley (Hordeum spontaneum) by BARE-1 retrotransposon dynamics in response to sharp microclimatic divergence. Proc. Natl. Acad. Sci, 2000;97, 6603-6607.

21. Boyko, E., Kalendar, R., Korzun, V., Gill, B., \& Schulman, A. H. Combined mapping of Aegilops tauschii by retrotransposon, microsatellite, and gene markers. Plant Mol. Biol, 2002;48, 767-790.

22. Teo, C. H., Tan, S. H., Ho, C. L., Faridah, Q. Z., Othman, R. Y., Heslop-Harrison, J. S., Kalendar, R., \& Schulman,
A. H. Genome constitution and classification using retrotransposon-based markers in the orphan crop banana. Journal of Plant Biology, 2005;48, 96-105.

23. Felsenstein, J. (2004). PHYLIP (Phylogeny Inference Package) version 3.6. Distributed by the author. Department of Genome Sciences, University of Washington, Seattle.

24. Chan, Y. K. (2002). Breeding papaya for resistance to ringspot virus disease in Malaysia. Symposium 20 (S20): Citrus and other subtropical and tropical fruit crops: issues, advances and oppurtunities, Metro Toronto Convention Centre. pp. 98.

25. Voytas, D. F., Cummings, M. P., Konieczny, A., Ausubel, F. M., \& Rodermel, S. R. Copia-like retrotransposons are ubiquitous among plants. Proc. Natl Acad. Sci, 1992;89, 7124-7128.

26. Teo, C. H., Tan, S. H., Othman, R. Y., \& Schwarzacher, T. The Cloning of Ty1-copia-like Retrotransposons from 10 Varieties of Banana (Musa Sp.). J Biochem Mol Biol Biophys, 2002;6, 193-201.

27. Shirasu, K., Schulman, A. H., Lahaye, T., \& Schulzelefert, P. A contiguous $66 \mathrm{~Kb}$ barley DNA sequence provides evidence for reversible genome expansion. Genome Res, 2000;10, 908-915.

28. San Miguel, P., Tikhonov, A., Jin, Y. K., Motchoulskaia, N., Zakharov, D., Berhan, A. M., Springer, P. S., Edwards, K. J., Lee, M., Avramova, Z., \& Bennetzen, J. L. Nested retrotransposons in the intergenic regions of the maize genome. Science, 1996;274, 765-768.

29. Pearce, S. R., Stuart-Rogers, C., Knox, M. R., Kumar, A., Ellis, T. H. N., \& Flavell, A. J. Rapid isolation of plant Ty1-copia group retrotransposon LTR sequences for molecular marker studies. The Plant Journal, 1999;19(6), 711-717.

30. Weising, K., Nybom, H., Wolff, K., \& Meyer, W. (1995). DNA fingerprinting in plants and fungi. United States of America: CRC Press, Inc. 